\title{
INVENTARIO FORESTAL EN LA ESTACIÓN PINDO MIRADOR
}

\author{
Directora: \\ Carmen Luzuriaga'
}

Colaboradora:

Ing. Forestal Cristina Cuasapaz

Ayudante de campo:

Gloria Quichimbo 



\section{Resumen}

El presente proyecto se ejecutó en la “Estación Biológica Pindo Mirador” gracias al financiamiento de la Universidad Tecnológica Equinoccial (UTE). La zona de estudio constituyó los bosques de la Estación; se consideró como variable la pendiente del terreno. El diseño utilizado fue el muestreo en fajas que permitió captar mejor la variación de la población, debido a la heterogeneidad en su composición florística. El tamaño de cada parcela fue de 200 X 50 metros, lo que representa $1 \mathrm{Ha}$; la intensidad de muestreo fue del 5\%. Además se trabajó con subparcelas circulares para inventariar la regeneración natural de las especies.

Los resultados evidencian especies forestales de uso maderable y no maderable. Se propone considerar al bosque como un sistema biológico con usos múltiples: valor ambiental, ecológico, recreativo; así como un lineamiento general la regeneración natural como proceso natural que mantiene al bosque. Este inventario servirá de punto de partida para establecer estrategias de manejo del bosque y como un estudio que puntualiza la importancia de la Declaratoria de Bosque Protector a la zona de estudio de la Estación Biológica Pindo Mirador.

\section{Abstract}

This project was implemented in the "Biological Station Pindo Mirador" with funding from the Technological University Equator (UTE). The study area constituted the forests of the station was considered as the slope variable. The sampling design was used in strips which allowed better capture the population variation due to heterogeneity in its floristic composition. The size of each plot was $200 \mathrm{X} 50$ meters which is $1 \mathrm{Ha}$. The intensity of sampling was $5 \%$, and worked with circular subplots, to inventory the natural regeneration ofspecies. The results show forest species and non-timber use, is proposed to consider the forest as a biological system with multiple uses: environmental value, ecological, recreational. As a general guideline natural regeneration as a natural process that keeps the forest. This inventory will serve as a starting point for establishing forest management strategies and a study that points out the importance of the Declaration of Protective Forest in the study area of the Biological Station Pindo Mirador.

Palabras clave: Datos dendrométricos, Clase diamétrica, Índice de valor de importancia, Fuente semillera, Posición sociológica.

Recibido: Mayo 2011

Aprobado: Julio 2011 


\section{INTRODUCCIÓN}

La presente investigación se realizó en los bosques de la Estación Biológica Pindo Mirador ubicados en la Alta Amazonía de la provincia de Pastaza; la singularidad de los bosques se evidenció en la diversidad, riqueza y uso de los árboles nativos presentes en pendiente alta, media y baja; variable bajo la cual se definió el diseño muestral.

Se realizó un relevamiento forestal con el objetivo de contribuir al conocimiento del bosque piemontano; se estableció lineamientos generales de manejo mediante la caracterización de la vegetación identificando clases diamétricas; se estableció las potencialidades del bosque como: fuente semillera y de recuperación a través del manejo de la regeneración natural.

El muestreo se utilizó como un procedimiento para la medición del bosque. El diseño de parcelas en franjas permitió cubrir la mayor cantidad de árboles, así como también se trabajó con parcelas circulares para cubrir la regeneración natural.

El interés de los bosques piemontanos se evidenció en los resultados. Las especies forestales no solo tienen un uso maderable sino que además ayudan a mantener la fauna, controlan la erosión del suelo, evitan desequilibrios climáticos, proporcionan servicios recreativos, mantienen la humedad, regulan el equilibrio hídrico y proveen productos naturales.

Este estudio propone considerar al bosque como un sistema biológico con propósitos múltiples; el valor ecológico, ambiental, biológico y recreativo constituye una fuente importante para el mantenimiento de la vida silvestre.

La preservación del bosque se plantea como uno de los lineamientos generales para el manejo sustentable del mismo y la regeneración natural como un proceso in situ que ayuda a mantener el equilibrio natural y garantiza el mantenimiento y recuperación del bosque secundario.

Los bosques de la Estación guardan mucha importancia por el valor que tienen las especies por lo que se requiere continuar con estudios de este tipo que contribuyan al conocimiento de la riqueza que tiene la Amazonía ecuatoriana. Y esta información valide y consolide la declaratoria de Bosque Protector y permita enfocarse al futuro con nuevas líneas de investigación.

\section{OBJETIVOS}

\section{Objetivo General}

Contribuir al conocimiento del Bosque piemontano y establecer lineamientos generales de manejo para la declaratoria de bosque protector, mediante la caracterización de la vegetación existente en la Estación Biológica Pindo Mirador.

\section{Objetivos específicos}

- Identificar la estructura vertical y horizontal del recurso forestal existente en la Estación Biológica Pindo Mirador.

- Establecer las potencialidades del Bosque Tropical como fuente semillera y las posibilidades de recuperación a través del manejo de la regeneración natural.

- Proponer Lineamientos Generales para el Manejo Sustentable del Bosque Amazónico.

- Determinar las especies forestales maderables y no maderables de acuerdo a la categoría de uso.

- Establecer parcelas permanentes de muestreo para posteriores estudios de dinámica forestal y biológica. 


\section{MATERIAL Y MÉTODOS}

\section{Área de estudio}

El área de estudio se ubica en Pastaza, considerada la provincia más grande del Ecuador. Limita al norte con las provincias Napo y Orellana; al sur con Morona Santiago; al este con Perú y al oeste con la provincia Tungurahua.

Específicamente se ubica en el cantón Mera, Colonia Pindo Mirador donde se encuentra la Estación Biológica Pindo Mirador en el Km 14, Vía Puyo - Baños. Se sitúa a una altitud de 1153 m.s.n.m. con unas coordenadas de $17^{\circ} 82>48^{\prime \prime}$ W y $98^{\circ} 38>67^{\prime \prime} \mathrm{N}$.

La Estación Biológica cuenta con un área de 274 hectáreas en la cual se encuentra la cuenca alta del río Pindo Grande y la cuenca del río Plata con numerosos riachuelos y afluentes. En cuanto al clima, es constante, oscila entre los $20-25^{\circ} \mathrm{C}$; se define como un clima mesotérmico, perhúmedo y de permanente lluvia, con precipitaciones medias anuales de $4500 \mathrm{~mm}^{3}$.

El tipo de vegetación corresponde a Bosque siempreverde piemontano de acuerdo a la clasificación realizada por Rodrigo Sierra (1999). La irregularidad de la topografía es evidente, las permanentes lluvias y la alta humedad facilitan derrumbes y la caída de árboles gigantescos.

\section{Material y métodos}

El material y equipos utilizados fueron los siguientes:

- GPS

- Cámara digital

- Flexómetro

- Binoculares

- Brújula

- Podadora aérea

- Podadora manual

- Libretas de campo

- Sogas

- Arnés

- Cinta métrica

- Placas metálicas

- Clavos

- Marcadores permanentes

- Fundas plásticas y de papel

- Machetes

- Pintura y brocha

\section{METODOLOGÍA}

Para realizar el levantamiento de datos en el bosque se utilizó el muestreo estadístico por la aplicación que presenta para estudios de este tipo, partiendo que la variabilidad es una característica de los bosques de la Amazonía.

La elección del diseño de muestreo se basó tomando en cuenta bibliografía especializada y los recursos disponibles, "la simplicidad de los diseños de muestreo puede dar buenas y efectivas operaciones de inventario."

El diseño utilizado fue el muestreo en fajas, "procedimiento muy popular en trabajos de inventarios de bosques naturales tropicales y subtropicales" ${ }^{2}$. Para esta investigación de inventario forestal de acuerdo a ThorstenJolitz(2001) en el Manual para estudios científicos recomienda que sean parcelas en franjas de tamaño equivalente, pues captan mejor la variación de la población, por lo que se adaptó a la realidad de los bosques de la Estación Biológica, los cuales presentan una heterogeneidad en su composición florística. El tamaño de cada parcela fue de $200 \times 50$ metros, lo que representa $1 \mathrm{Ha}$.

1 Jaramillo, C. (2006) Inventario Forestal. Pág. 17

$2 \quad$ Ibid. Pag. 74 


\section{Intensidad del muestreo}

La distribución de las parcelas se localizó en diferente tipo de pendiente alta, media y baja. Se consideró la superficie aproximada que existe de bosque de acuerdo a la cobertura boscosa en la Estación Biológica, la intensidad de muestreo fue del 5\% y de allí se estableció el número de parcelas. Dentro de cada parcela se localizó una subparcela circular de 2 m de diámetro, para inventariar la regeneración natural de las especies.

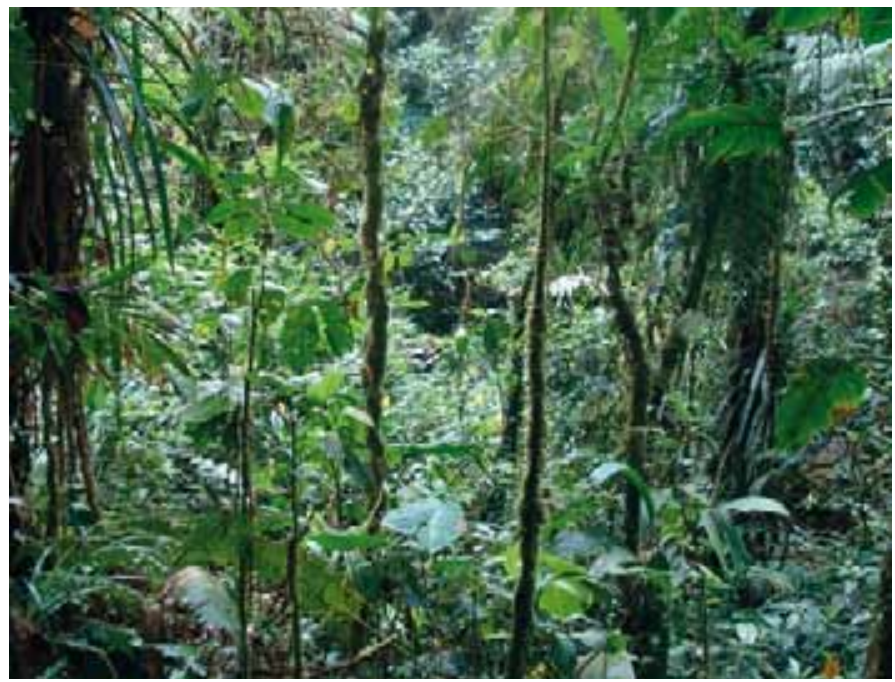

Foto 1. Área de estudio en la Estación Biológica Pindo Mirador

Fuente: Investigación directa

Elaboración: Carmen Luzuriaga

\section{Cálculo de parcelas en el bosque}

El cálculo del número de parcelas que se establecieron se determinó con base al área de la Estación Biológica que constituyen $274 \mathrm{Ha}$, tomando en cuenta para este estudio el 5\% para la muestra. \# Parcelas= $274 \times 5 \% / 100 \%=13,7$ redondeando 14 parcelas.

\section{Distribución de las parcelas de acuerdo a la cobertura boscosa}

La Estación Biológica de acuerdo a la cobertura boscosa posee Bosque Primario y Bosque Secundario. Las parcelas se distribuyeron de la misma forma estimando el $5 \%$ de acuerdo al porcentaje en cada tipo de bosque existente.
Cuadro 1. Composición del bosque

\begin{tabular}{|l|l|l|}
\hline Tipo de Bosque & Ha & $\%$ \\
\hline Primario & 55 & 20 \\
\hline Secundario & 192 & 70 \\
\hline Areas Alteradas & 27 & 10 \\
\hline Total & 274 & 100 \\
\hline
\end{tabular}

Fuente: Investigación Directa

Elaboración: Carmen Luzuriaga

Cuadro 2. Número de parcelas en Bosque

\begin{tabular}{|l|l|l|}
\hline Tipo de Bosque & F & N \\
\hline Primario & 55 & 3 \\
\hline Secundario & 192 & 10 \\
\hline Areas Alteradas & 27 & 1 \\
\hline Total & 274 & 100 \\
\hline
\end{tabular}

Fuente: Investigación Directa

Elaboración: Carmen Luzuriaga

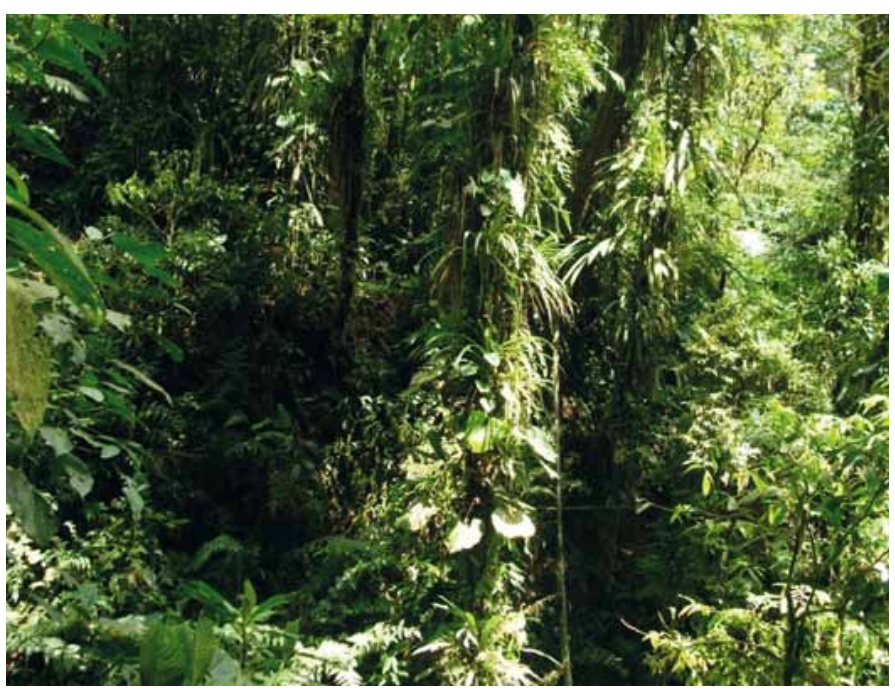

Foto 2. Parcela en Bosque Primario.

Fuente: Investigación Directa

Elaboración: Carmen Luzuriaga

\section{Delimitación de parcelas}

Para delimitar las parcelas se utilizó tubos PVC, los cuales se colocaron en las esquinas de las parcelas. 


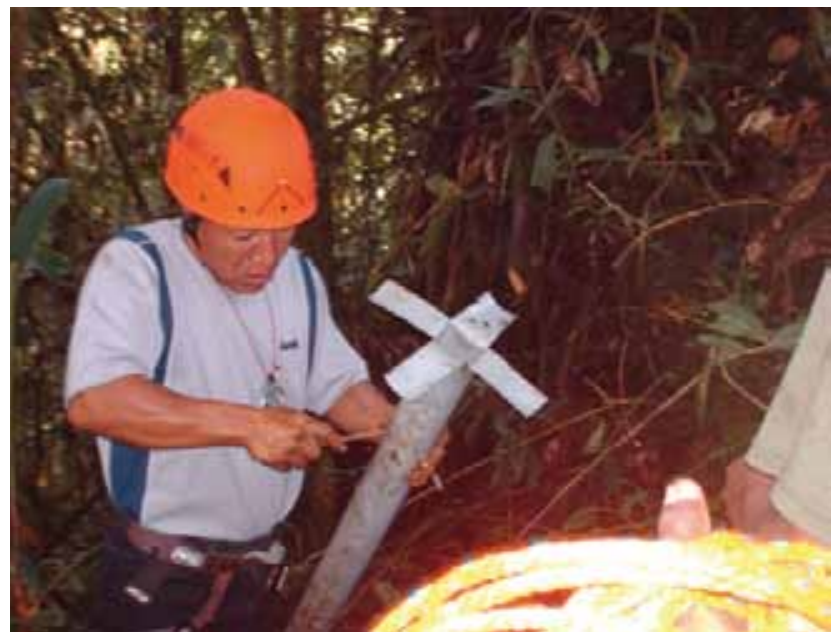

Foto 3. Colocando varilla en la base del tubo.

Fuente: Investigación Directa

Elaboración: Carmen Luzuriaga

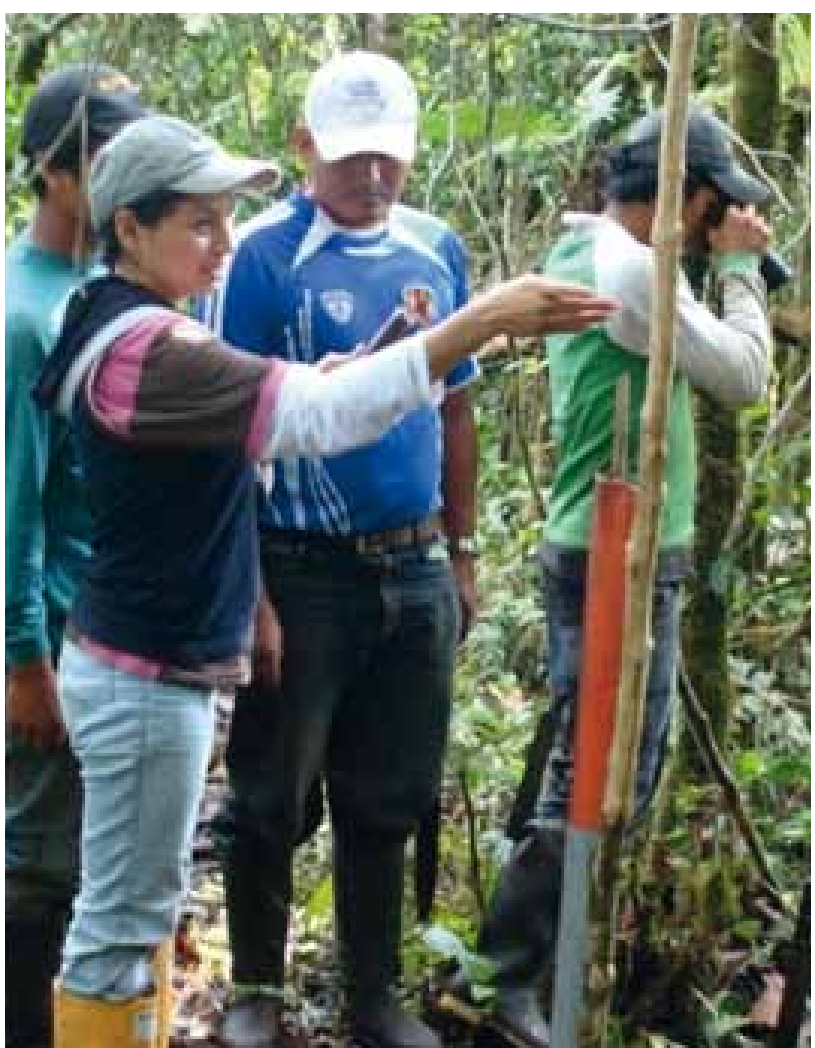

Foto 4. Supervisando las parcelas permanentes.

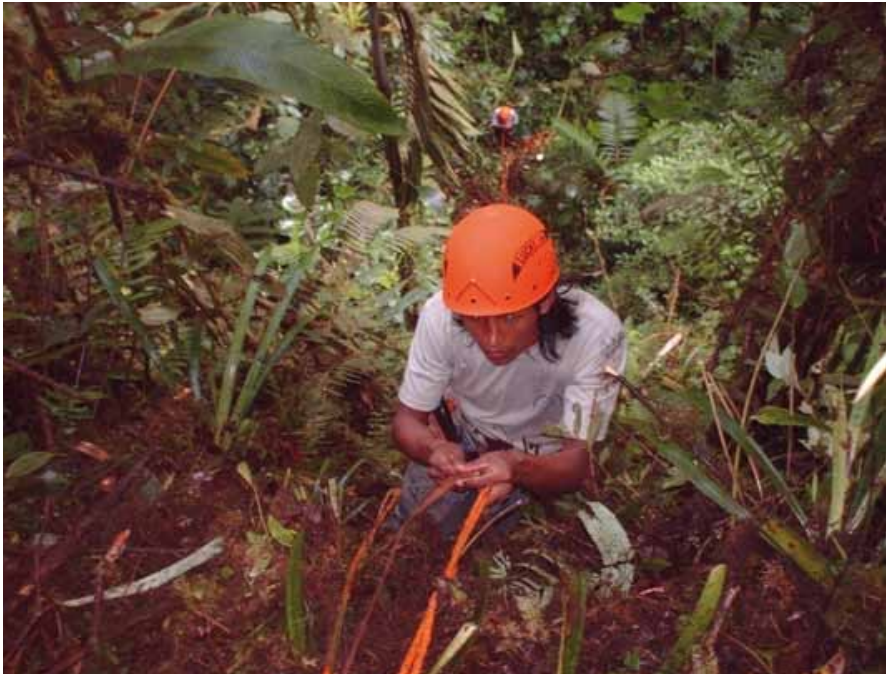

Foto5. Trabajador bajando con arnés en

Bosque Primario pendiente alta.

Fuente: Investigación Directa

Elaboración: Carmen Luzuriaga

\section{Toma de datos}

Una vez definida la parcela se procedió al inventario total de especies forestales que son el objetivo de estudio. Los parámetros para el registro de datos fueron los siguientes: Nombre Común, DAP, Altura, Regeneración, Fuste, Posición Sociológica.
a. NOMBRE COMÚN
b. DIÁMETRO A LA ALTURA DEL PECHO
c. ALTURA TOTAL
d. REGENERACIÓN
e. FUSTE

Se consideró 4 categorías:

1=Recto, 2= Ligeramente torcido, 3= Torcido y 4= Muy torcido.

f. POSICIÓN SOCIOLÓGICA

Fuente: Investigación Directa

Elaboración: Carmen Luzuriaga 


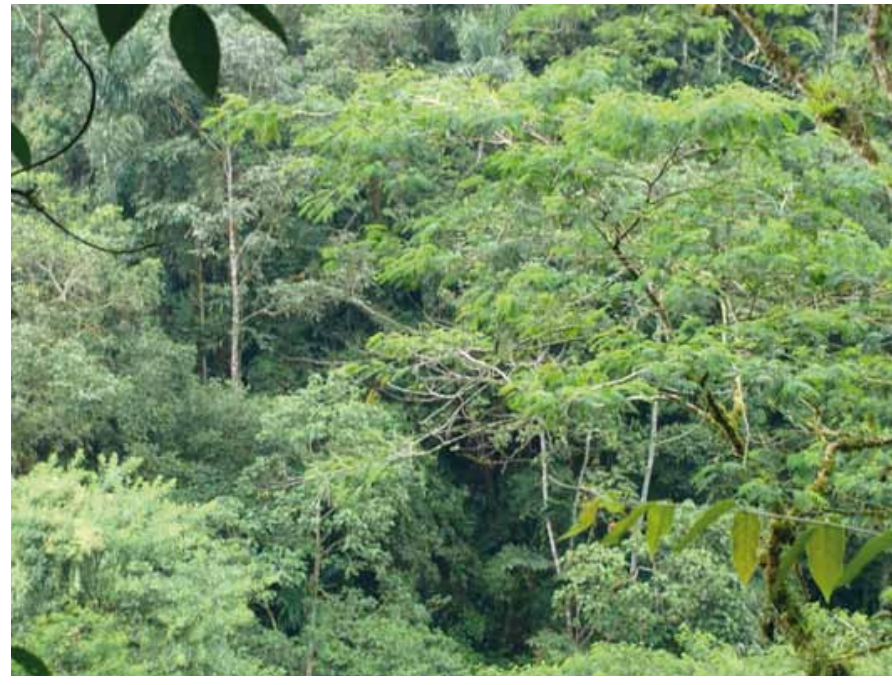

Foto 6. Dosel del bosque primario.

Fuente: Investigación Directa

\section{Elaboración: Carmen Luzuriaga}

Se considera a los diferentes niveles o estratos en los cuales se puede dividir la estratificación vertical del bosque. Para determinar la posición sociológica se establecieron 3 estratos: Dosel, subdosel y sotobosque.

Dosel.- aquellos árboles cuya altura total sea igual o mayor a los 11 metros.

Subdosel.- aquellos árboles cuya altura total oscile entre los 6 y 10 metros.

Sotobosque.- aquellos árboles cuya altura total sea igual o menor a 5 metros.

\section{IDENTIFICACIÓN DE ÁRBOLES}

La identificación de las especies forestales se realizó directamente en el bosque y de aquellos árboles que no pudieron ser identificados se colectó muestras. Éstas fueron enviadas al Herbario Nacional del Ecuador (QCNE) para su respectiva identificación.

\section{EVALUACIÓN DE CRITERIOS PARA PARCELAS PERMANENTES}

Las parcelas permanentes se establecieron tomando en cuenta criterios que permitan evaluar a futuro la dinámica y crecimiento del bosque. Se colocó placas metálicas en las diferentes especies de árboles para su localización e identificación.

Los datos y mediante una tabla Excel se han calculado el volumen de madera y el DAP (Diámetro a la Altura de Pecho).

$$
\text { Volumen }=p * r^{2}
$$

La diversidad se ha calculado mediante el índice de Shannon (H'). Para ello, en cada parcela la cobertura específica se ha pasado a porcentajes para tener una visión más concreta del territorio. Para hacer estos cálculos no se han tenido en cuenta la materia orgánica ni el humus, aunque cabe destacar que son muy abundantes. $\mathrm{H}^{\prime}=-\Sigma \mathrm{p}_{\mathrm{i}} \times \log _{2} \mathrm{p}_{\mathrm{i}}$; donde $\mathrm{p}_{\mathrm{i}}$ es la probabilidad de la especie i.

\section{RESULTADOS}

\section{Zonificación del área de estudio.}

El área de estudio se realizó con base al tipo de bosque y a la pendiente.

Cuadro 3. Zonificación del área de estudio

\begin{tabular}{|l|c|c|}
\hline \multirow{2}{*}{ BOSQUE } & \multicolumn{2}{|c|}{ No. De PARCELAS } \\
\cline { 2 - 3 } & PRIMARIO & SECUNDARIO \\
\hline PENDIENTE ALTA & 1 & 3 \\
\hline PENDIENTE MEDIA & 1 & 3 \\
\hline PENDIENTE BAJA & 1 & 4 \\
\hline
\end{tabular}

Fuente: InvestigaciónDirecta

Elaboración: Carmen Luzuriaga 


\section{BOSQUE SECUNDARIO}

\section{Estructura Vertical Bosque Secundario}

Cuadro 4. Zonificación del área de estudio

\begin{tabular}{|c|r|r|r|}
\hline \multirow{2}{*}{ BOSQUE SECUNDARIO } & \multicolumn{3}{|c|}{ ALTURAS(m) } \\
\cline { 2 - 4 } & Piso inferior & Piso medio & Piso superior \\
\cline { 2 - 4 } & $0-10$ & $10,1-20$ & $20,1-35$ \\
\hline PB & 1 & 285 & 257 \\
\hline PM & 5 & 168 & 52 \\
\hline PA & 1 & 274 & 145 \\
\hline
\end{tabular}

Fuente: InvestigaciónDirecta

Elaboración: Carmen Luzuriaga

El mayor número de individuos (Cuadro 4) se encuentra en el piso medio con 285 individuos en pendiente baja, 168 en pendiente media y 274 en pendiente alta.

\section{Estructura horizontal (diámetros) Bosque Secundario}

Cuadro 5. Distribución Diamétrica en Bosque Secundario.

\begin{tabular}{|l|r|r|r|r|r|r|}
\hline \multirow{2}{*}{ BOSQUE } & \multicolumn{7}{|c|}{ CLASES DIAMETRICAS $(\mathrm{cm})$} \\
\cline { 2 - 7 } & $0 \mathrm{~cm}-$ & $20,1 \mathrm{~cm}-$ & & & & \\
& $20 \mathrm{~cm}$ & $30 \mathrm{~cm}$ & $30,1 \mathrm{~cm}-40 \mathrm{~cm}$ & $40,1 \mathrm{~cm}-50 \mathrm{~cm}$ & $50,1 \mathrm{~cm}-60 \mathrm{~cm}$ & $>60,1 \mathrm{~cm}$ \\
\hline PB & 4 & 455 & 84 & 13 & 1 & 3 \\
\hline PM & 0 & 149 & 56 & 14 & 5 & 1 \\
\hline PA & 1 & 301 & 82 & 21 & 9 & 6 \\
\hline
\end{tabular}

Fuente: InvestigaciónDirecta

Elaboración: Carmen Luzuriaga

El mayor número de individuos (Cuadro 5) se presenta en las clases diamétricas de $20,1 \mathrm{~cm}$ a $30 \mathrm{~cm}$ con 455 individuos para parcelas con pendiente baja, 149 en parcelas de pendiente media y 301 en parcelas de pendiente alta.

Índice de Valor de Importancia, Abundancia y Dominancia en Bosque Secundario.
En bosque secundario el pigue (Piptocomadiscolor), es la especie que presenta los valores más altos de dominancia en los tres tipos de pendiente consideradas en este estudio. En pendiente baja (Cuadro 6) presenta valores del 34,17\% con 315 individuos y el IVI de $41,67 \%$. En pendiente media(Cuadro 7) tiene valores de 30,88\% en dominancia con 91 individuos y un valor de IVI de 64,169\%. En pendiente alta (cuadro 8) tiene un valor de $21,12 \%$ con 158 individuos y un valor de IVI de 43,91\%.

\section{Regeneración Natural en Bosque Secundario.}

En el bosque secundario (Cuadro 9), se encontró 26 especies con 194 individuos en pendiente baja, 35 especies con 175 individuos en pendiente media y 31 especies con 154 individuos en pendiente alta.

Las especies con mayor regeneración son: Canelo (Ocotealeucoxylon)49, Colca (Miconiasplendens) (42), Yacu caspi (Lozaniaklugii)

\section{BOSQUE PRIMARIO}

Estructura Vertical de Bosque Primario.

Cuadro 11. Estructura vertical en Bosque Primario.

\begin{tabular}{|l|r|r|r|}
\hline \multicolumn{4}{|c|}{ ALTURAS (m) } \\
\hline BOSQUE & $0-10$ & $10,1-20$ & $20,1-35$ \\
PRIMARIO & 0 & 98 & 33 \\
\hline PB & 5 & 106 & 56 \\
\hline PM & 0 & 108 & 22 \\
\hline PA & 5 & 312 & 111 \\
\hline TOTAL & & &
\end{tabular}

Fuente: Investigación Directa

Elaboración: Carmen Luzuriaga 
En el cuadro 11 se observa que el mayor número de individuos se encuentra en el piso medio con 108 individuos en pendiente alta, 106 en pendiente media y 98 en pendiente baja.

\section{Estructura Horizontal en Bosque Primario.}

Cuadro 12. Distribución Diamétrica en Bosque Primario.

\begin{tabular}{|l|r|r|r|r|r|r|}
\hline \multicolumn{7}{|c|}{ CLASES DIAMETRICAS (cm) } \\
\hline $\begin{array}{l}\text { BOSQUE } \\
\text { PRIMARIO }\end{array}$ & $0-20$ & $20,1-30$ & $30,1-40$ & $40,1-50$ & $50,1-60$ & $>60,1$ \\
\hline PB & & 89 & 28 & 8 & 2 & 4 \\
\hline PM & & 94 & 37 & 22 & 6 & 8 \\
\hline PA & & 70 & 42 & 9 & 8 & 1 \\
\hline TOTAL & 0 & 253 & 107 & 39 & 16 & 13 \\
\hline
\end{tabular}

Fuente: InvestigaciónDirecta

Elaboración: Carmen Luzuriaga

El mayor número de individuos se encuentra en las clase diamétrica de 20,1 a $30 \mathrm{~cm}$ con valores de 89, 94 y 70 en pendiente baja, media y alta respectivamente.

Índice de Valor de Importancia, Abundancia y Dominancia en Bosque Primario.

En Bosque Primario se registra como especie dominante el Ponce (Alchornea latifolia)en los tres tipos de pendiente. En pendiente baja (Cuadro 13), registra un valor muy superior en dominancia con $36,07 \%$, con 52 individuos y un IVI de 75,77\%. En pendiente media (Cuadro 14), la especie tiene una dominancia de 34 individuos, con un porcentaje de $19,11 \%$ y un IVI de 39, 46\%. En pendiente alta (Cuadro 15), con 16 individuos, con un porcentaje del 11,46\% y un IVI de 23,77.

\section{Regeneración Natural en Bosque Primario.}

Las especies con mayor regeneración son Arrayan (Farameauniflora) con 31 individuos, Canelo (Ocotealongifolia) 14, Colca (Miconiatrinervia)con 18, Guabilla (Zygiacoccinea) con 9 y Guarango (Macrolobium acaciafolium) con 11 individuos.

\section{Especies Forestales Maderables y No Maderables Especies Forestales Maderables}

En el cuadro 17 se evidencia que entre las especies maderables de buena calidad se encuentran las siguientes: Cedro (Cedrela odorata), Copal (Protiumamazonicum), Laurel (Cordia alliodora), Canelo amarillo (Ocotealeucoxylon), Doncel (Otoba parvifolia) y Pumamaqui (Oreopanaxpalamophyllus).

EspeciescomoelPigue(Piptocomadiscolor), Canelo(Endlicheria sp.),Achotillo (Vismia lateriflora), Roble (Ardisiaguianensis), Hintachi (Joosiaumbellifera), Ortiguillo (Perebeasp.) son especies de madera corriente.

\section{Especies Forestales No Maderables}

Los árboles nativos de la Amazonía presentan gran utilidad en la supervivencia de la gente que utiliza diferentes partes del árbol para curar ciertas enfermedades, como medicinas naturales, frutos, semillas para elaboración de collares, adornos o recuerdos.

A continuación se mencionan algunos usos de especies no maderables:

Látex y resinas: Sangre de drago (Crotonlechleri), para cicatrizar heridas; Copal (Protiumamazonicum), para dolores reumáticos; Sande (Brosimumutile), dolor de estómago.

Frutos: Guabas. (Inga sp.) Sirven de alimento para animales silvestres. Melliso (Orsomiacoccinea), las semillas se utiliza para collares.

Ornamentales: (Sennabacillaris), arbustos con vistosas flores amarillas y además insectos. 


\section{DISCUSIÓN}

De acuerdo al estudio realizado en los bosques de la Estación Biológica Pindo Mirador se estableció tres estratos superior (dosel), medio (subdosel) e inferior. El mayor número de árboles y de especies se encuentra en el estrato medio y el menor número de especies corresponde a los estratos superior e inferior. Encontrándose las especies jóvenes en bosque primario y secundario en una etapa de sucesión luego de haber existido una alteración en su hábitat natural. Los resultados indican que existen tres especies con un alto valor de importancia (IVI), la mayor dominancia y abundancia presentaron las siguientes especies: Piptocomadiscolor (Pigue), Alchornea latifolia (Ponce) y Brunelliastenoptera (Malva).

Como lineamiento general para el Manejo Sustentable del Bosque implica considerar el valor ecológico, biológico, ambiental que tiene el bosque al mantener como prioridad la preservación. Es importante preservar los bosques "la preservación suele impedir el aprovechamiento de especies o ecosistemas"3, El objetivo de la preservación de especies y ecosistemas es garantizar su continuidad. Se puede considerar como un lineamiento de manejo el establecer a los bosques "como sistemas biológicos con propósitos múltiples"; los recursos forestales ayudan a mantener la fauna, controlan la erosión del suelo, evitan desequilibrios climáticos, proporcionan servicios recreativos, atraen la lluvia, mantienen la humedad, regulan el equilibrio hídrico de los ríos, proveen productos naturales. Los servicios ambientales y recreativos proporcionados por el bosque nunca decrecen con la edad, siempre que no alcance el marchitamiento o decrepitud de la masa. Los valores recreativos y estéticos constituyen una fuente importante de apoyo para el mantenimiento de las especies silvestres.

3 Nebel, B. Ciencias ambientales. Ecología y desarrollo sostenible Pág. 496.

4 Romero, C. Economía de los Recursos ambientales y naturales. Pág. 147.
El manejo del bosque en la Estación Biológica Pindo Mirador debe continuar con los procesos de regeneración natural. Esto a pesar de que las especies forestales maderables han sido explotadas existiendo muy pocas, por lo que es necesario realizar raleos, anillamiento y liberación, como técnicas que ayudan al crecimiento de otras especies forestales importantes.

Al considerar la potencialidad del bosque piemontano como fuente semillera se evidenció que las características fenológicas no son buenas debido al aprovechamiento forestal que existió años atrás o explotación; sin embargo, la única especie forestal que está en condiciones de ser considerada es el Pigüe.

\section{CONCLUSIONES}

\section{BOSQUE SECUNDARIO}

- De los resultados obtenidos y según la clasificación simple de la estructura vertical del vuelo de lufro (1979) presente en Lamprecht (1981) en la que se distingue: piso superior (altura > $2 / 3$ de la altura superior del vuelo de $20,1 \mathrm{~m}$ a $35 \mathrm{~m}$ ), piso medio $(<2 / 3>1 / 3$ de $10,1 \mathrm{~m}$ a $20 \mathrm{~m})$ y piso inferior $(<1 / 3$ de la altura superior del vuelo de o a 10m ) el mayor número de individuos se encuentra dentro del piso medio o sub dosel 10,1m a $20 \mathrm{~m}$. con 274 individuos, por lo que es un bosque joven.

- De acuerdo a los diámetros de las especies se obtuvo que existe la mayor dominancia de individuos en la clase diamétrica de $20,1 \mathrm{~cm}$ a $30 \mathrm{~cm}$. encontrando el $73,05 \%$ de especies.

- Las especies de Pigue, Ponce y Malva por ser especies heliófilas de rápido crecimiento se encontraron en mayor número ya que sus condiciones encontradas para el desarrollo fueron las apropiadas después del aprovechamiento del bosque que se realizó en años anteriores.

- Las especies forestales tienen mejores condiciones para su desarrollo y regeneración en áreas con pendiente baja y media.

- Las especies de buena calidad de madera y con el menor 
número de individuos son: Canelo, Copal, Doncel, Cedro, Sande, Sangre de gallina, Tucuta, Motilon.

- No fue posible identificar árboles semilleros debido a que no se encontraron el número de especies óptimas en la zona con buenas características fenológicas.

\section{BOSQUE PRIMARIO}

- De los resultados obtenidos y según la clasificación simple de la estructura vertical del vuelo de lufro (1979) presente en Lamprecht (1981) en la que se distingue: piso superior (altura > $2 / 3$ de la altura superior del vuelo de $20,1 \mathrm{~m}$ a $35 \mathrm{~m}$ ), piso medio $(<2 / 3>1 / 3$ de $10,1 \mathrm{~m}$ a $20 \mathrm{~m})$ y piso inferior $(<1 / 3$ de la altura superior del vuelo de o a 10m ) el bosque se encuentra dentro del piso medio $10,1 \mathrm{~m}$ a $20 \mathrm{~m}$ con 312 individuos superando en 100\% al piso superior de 111 individuos, se calificaría dentro del sub dosel o piso medio.

- El mayor número de individuos se encuentra dentro de la clase diamétrica de $20,1 \mathrm{~cm}$ a $30 \mathrm{~cm}$ con 253 individuos lo que representa más de 50\% de la población total.

- Dentro del bosque primario se observa una marcada diferencia con la especie Ponce en cuanto a número de individuos y dominancia ya que supera en un $75 \%$ a los valores de las otras especies.

- El bosque en estudio es heterogéneo por su gran diversidad que existe obteniendo 40 especies registradas en 1 ha.

- Las especies de Cedro, Copal, Doncel, Laurel, Ceibo tienen 1 individuo por ha.

- Las especies de mayor regeneración natural son Colca, Canelo, Guarango y Arrayan

\section{RECOMENDACIONES}

- Los resultados obtenidos confirman el estado de conservación de las diferentes especies en el bosque pero es necesario considerar para estudios posteriores factores edáficos, exposición al viento, la presencia de fauna silvestre y otros que inciden directamente en la diversidad y riqueza florística.

- Se debería reforestar el área con especies que tienen mejor regeneración y desarrollo.

- Realizar tratamientos silviculturales como liberación, anillamiento para ayudar al mejor desarrollo de las especies de madera buena y media.

- Realizar ensayos de germinación y crecimiento de especies de poca regeneración, de importante valor ecológico y maderable como es el caso del Sande, Cedro, Laurel. etc.

- Realizar enriquecimiento del bosque con especies maderables. 


\section{BIBLIOGRAFÍA}

Añazco, Marío.(sf).Productos Forestales no madereros en el Ecuador. DFC/Ministerio de Ambiente/Gobierno de los Países Bajos. Quito.

Cerón, C.E. (1993). Impactos sobre la vegetación en áreas naturales del Ecuador.

Cerón, Carlos. (1998). Etnobotánica de los Huaorani de Quehueiri-ono Napo-Ecuador. Herbario Alfredo Paredes. Universidad Central del Ecuador. Quito.

Cerón, C.E. (2003). Manual de botánica, sistemática, etnobotánica y métodos de estudio en el Ecuador. Herbario Alfredo Paredes (QAP, Escuela de Biología de la Universidad Central del Ecuador. Editorial Universitaria, Quito.

Lozano, P. \& D. Medranda. (2008). Plan de Manejo y Estudios Iniciales de Flora y Fauna del Bosque Protector Abanico.Hidroabanico S.A. \& Fundación de Investigación y Asistencia Social.

Luzuriaga, Carmen. (2007). Diagnóstico de la Flora Estación Biológica Pindo Mirador. CODEU. Quito.

Monserrat, M.J. Koizol, H. Borgtoft Pedersen \&Granda. (2007). Plantas Útiles del Ecuador. Ediciones Abya-Yala. Quito- Ecuador.

Morocho, D. \& J. C. Romero. (2003). Bosques del Sur. El estado de 12 remanentes de bosques andinos de la provincia de Loja. FundaciónEcológica Arco Iries/PROBONA/DICA. Loja.

Nebel, Bernard. (1999). Ciencias ambientales. Ecología y desarrollo sostenible. PRENTICE HALL. México.

Neill, David \& W. Palacios. (1991). Curso de Dendrología en la Amazonía Ecuatoriana. Estación Biológica Jatun Sacha. Miashualli. NapoEcuador.

Romero, Carlos. (1994). Economía de los Recursos ambientales y naturales. Editorial Alianza. Madrid.
Sierra, Rodrigo. (1999). Propuesta Preliminar de un Sistema de Clasificación de Vegetación para el Ecuador Continental. Proyecto INEFAN/GEF-BIRFy EcoCiencia. Quito-Ecuador.

Thorsten, Jolitz. (2001). Manual para estudios científicos en Forestería, Agricultura y Ecología. Fundación Jatun Sacha. Quito.

Vásquez, Rodolfo \& Rocío Rojas. (2003). Plantas de la Amazonía Peruana. Missouri Botanical Garden. 


\section{ANEXOS}

Cuadro 6. Bosque Secundario Pendiente Baja.

\begin{tabular}{|c|c|c|c|c|c|c|c|c|}
\hline NOMBRE COMÚN & Suma de F & $\begin{array}{c}\text { Suma de } \\
\qquad A B\end{array}$ & $\begin{array}{c}\text { Suma de } \\
\text { DR }\end{array}$ & $\%$ & $\begin{array}{c}\text { Suma de } \\
\text { DM }\end{array}$ & $\%$ & $\begin{array}{c}\text { Suma de } \\
\text { IVI }\end{array}$ & $\%$ \\
\hline ACHOTILLO & 7 & 0,315 & 6,090 & 1,500 & 4,950 & 0,763 & 11,040 & 1,046 \\
\hline BALSA BLANCA & 2 & 0,090 & 2,439 & 0,601 & 1,327 & 0,204 & 3,766 & 0,357 \\
\hline Boyablanca & 1 & 0,059 & 0,448 & 0,110 & 0,414 & 0,064 & 0,862 & 0,082 \\
\hline CANELO AMARILLO & 4 & 0,287 & 4,878 & 1,201 & 72,175 & 11,118 & 77,053 & 7,302 \\
\hline CANELO BLANCO & 3 & 0,300 & 2,977 & 0,733 & 4,260 & 0,656 & 7,237 & 0,686 \\
\hline CARA HUASCA & 1 & 0,056 & 1,220 & 0,300 & 0,831 & 0,128 & 2,050 & 0,194 \\
\hline CEDRILLO & 1 & 0,057 & 1,220 & 0,300 & 0,851 & 0,131 & 2,070 & 0,196 \\
\hline CEIBO & 1 & 0,137 & 0,448 & 0,110 & 0,960 & 0,148 & 1,408 & 0,133 \\
\hline COLCA DE BP & 2 & 0,068 & 2,439 & 0,601 & 1,011 & 0,156 & 3,450 & 0,327 \\
\hline COLCA HOJA FINA & 1 & 0,041 & 0,448 & 0,110 & 0,290 & 0,045 & 0,738 & 0,070 \\
\hline COPAL & 1 & 0,036 & 1,220 & 0,300 & 0,529 & 0,081 & 1,748 & 0,166 \\
\hline DONCEL COCO & 1 & 0,086 & 1,220 & 0,300 & 1,274 & 0,196 & 2,493 & 0,236 \\
\hline DRAGO & 27 & 1,414 & 12,464 & 3,069 & 10,613 & 1,635 & 23,077 & 2,187 \\
\hline ESPONJA & 3 & 0,104 & 4,348 & 1,071 & 3,273 & 0,504 & 7,621 & 0,722 \\
\hline GUABA & 1 & 0,076 & 0,448 & 0,110 & 0,537 & 0,083 & 0,986 & 0,093 \\
\hline GUABA BEJUCA & 11 & 1,164 & 13,415 & 3,303 & 198,482 & 30,573 & 211,896 & 20,079 \\
\hline GUABILLA DE & & & & & & & & \\
\hline MONTE & 11 & 0,608 & 13,415 & 3,303 & 8,999 & 1,386 & 22,413 & 2,124 \\
\hline GUARANGO & 4 & 0,211 & 4,878 & 1,201 & 3,119 & 0,480 & 7,997 & 0,758 \\
\hline GUARUMO & 14 & 0,704 & 8,591 & 2,116 & 6,134 & 0,945 & 14,726 & 1,395 \\
\hline HABIO & 1 & 0,458 & 1,220 & 0,300 & 6,782 & 1,045 & 8,002 & 0,758 \\
\hline MALVA & 106 & 6,270 & 55,270 & 13,610 & 55,661 & 8,574 & 110,931 & 10,512 \\
\hline MARIA & 1 & 0,039 & 1,220 & 0,300 & 0,577 & 0,089 & 1,796 & 0,170 \\
\hline MATA PALO & 1 & 0,059 & 1,220 & 0,300 & 0,871 & 0,134 & 2,090 & 0,198 \\
\hline ORTIGUILLO & 6 & 0,365 & 13,415 & 3,303 & 5,407 & 0,833 & 18,821 & 1,784 \\
\hline PIGUE & 315 & 17,460 & 217,937 & 53,666 & 221,876 & 34,177 & 439,814 & 41,677 \\
\hline PONCE & 30 & 2,382 & 29,875 & 7,357 & 26,758 & 4,122 & 56,634 & 5,367 \\
\hline UVA & 1 & 0,054 & 0,448 & 0,110 & 0,376 & 0,058 & 0,825 & 0,078 \\
\hline UVA DE MONTE & 2 & 0,720 & 2,439 & 0,601 & 10,650 & 1,641 & 13,089 & 1,240 \\
\hline SIN NOMBRE & 1 & 0,031 & 0,448 & 0,110 & 0,215 & 0,033 & 0,663 & 0,063 \\
\hline \begin{tabular}{|l|} 
Total general \\
\end{tabular} & 560 & 33,652 & 406,098 & 100,000 & 649,201 & 100,000 & 1055,299 & 100,000 \\
\hline
\end{tabular}

Fuente: InvestigaciónDirecta

Elaboración: Carmen Luzuriaga 
Cuadro 7. Bosque Secundario Pendiente Media.

\begin{tabular}{|c|c|c|c|c|c|c|c|c|}
\hline NOMBRE COMÚN & Suma de $F$ & $\begin{array}{l}\text { Suma de } \\
A B\end{array}$ & $\begin{array}{l}\text { Suma } \\
\text { de DR }\end{array}$ & $\%$ & $\begin{array}{l}\text { Suma } \\
\text { de DM }\end{array}$ & $\%$ & $\begin{array}{l}\text { Suma } \\
\text { de IVI }\end{array}$ & $\%$ \\
\hline Achotillo & 13 & 0,428 & 13,665 & 4,555 & 6,848 & 2,283 & 20,513 & 6,838 \\
\hline Achotillo macho & 2 & 0,063 & 2,121 & 0,707 & 1,102 & 0,367 & 3,224 & 1,075 \\
\hline Balsa blanca & 1 & 0,032 & 1,010 & 0,337 & 0,501 & 0,167 & 1,511 & 0,504 \\
\hline Canelo amarillo & 1 & 0,051 & 0,735 & 0,245 & 0,514 & 0,171 & 1,249 & 0,416 \\
\hline Canelo blanco & 3 & 0,163 & 2,206 & 0,735 & 1,641 & 0,547 & 3,847 & 1,282 \\
\hline Canelo negro & 2 & 0,074 & 2,020 & 0,673 & 1,168 & 0,389 & 3,188 & 1,063 \\
\hline Canelo olor a cereza & 1 & 0,067 & 1,111 & 0,370 & 1,282 & 0,427 & 2,393 & 0,798 \\
\hline Caracaspi & 1 & 0,039 & 0,735 & 0,245 & 0,394 & 0,131 & 1,129 & 0,376 \\
\hline Caucho & 2 & 0,119 & 2,121 & 0,707 & 2,139 & 0,713 & 4,260 & 1,420 \\
\hline Cedrillo & 1 & 0,048 & 1,010 & 0,337 & 0,768 & 0,256 & 1,778 & 0,593 \\
\hline Chicle & 1 & 0,107 & 1,010 & 0,337 & 1,699 & 0,566 & 2,710 & 0,903 \\
\hline Colca & 12 & 0,156 & 13,333 & 4,444 & 2,968 & 0,989 & 16,302 & 5,434 \\
\hline Colcabp & 1 & 0,000 & 0,735 & 0,245 & 0,000 & 0,000 & 0,735 & 0,245 \\
\hline Colcaamarilla & 4 & 0,164 & 2,941 & 0,980 & 1,653 & 0,551 & 4,594 & 1,531 \\
\hline Colca de hoja ancha & 3 & 0,136 & 3,030 & 1,010 & 2,154 & 0,718 & 5,185 & 1,728 \\
\hline Colca de hoja fina & 4 & 0,157 & 4,040 & 1,347 & 2,485 & 0,828 & 6,525 & 2,175 \\
\hline Colcahoja roja & 1 & 0,037 & 1,010 & 0,337 & 0,584 & 0,195 & 1,594 & 0,531 \\
\hline Copal & 3 & 1,168 & 3,232 & 1,077 & 20,901 & 6,967 & 24,133 & 8,044 \\
\hline Doncel & 1 & 0,032 & 0,735 & 0,245 & 0,319 & 0,106 & 1,054 & 0,351 \\
\hline Drago & 2 & 0,126 & 1,846 & 0,615 & 1,665 & 0,555 & 3,511 & 1,170 \\
\hline Espuma & 1 & 0,098 & 0,735 & 0,245 & 0,989 & 0,330 & 1,725 & 0,575 \\
\hline Guababejuca & 5 & 0,233 & 5,152 & 1,717 & 3,797 & 1,266 & 8,948 & 2,983 \\
\hline Guababejuca de monte & 6 & 0,139 & 4,412 & 1,471 & 1,399 & 0,466 & 5,811 & 1,937 \\
\hline Guaba de río & 8 & 0,746 & 5,882 & 1,961 & 7,525 & 2,508 & 13,407 & 4,469 \\
\hline Guaba del río & 2 & 0,130 & 2,020 & 0,673 & 2,062 & 0,687 & 4,082 & 1,361 \\
\hline Guabilla & 5 & 0,232 & 5,152 & 1,717 & 3,835 & 1,278 & 8,987 & 2,996 \\
\hline Guabilla de monte & 2 & 0,564 & 1,471 & 0,490 & 5,690 & 1,897 & 7,161 & 2,387 \\
\hline Guabilla hoja fina & 2 & 0,097 & 1,471 & 0,490 & 0,978 & 0,326 & 2,448 & 0,816 \\
\hline Guarango & 5 & 0,296 & 3,676 & 1,225 & 2,988 & 0,996 & 6,664 & 2,221 \\
\hline Guarangorojo & 2 & 0,118 & 2,020 & 0,673 & 1,867 & 0,622 & 3,887 & 1,296 \\
\hline Guarumo & 2 & 0,083 & 2,121 & 0,707 & 1,428 & 0,476 & 3,549 & 1,183 \\
\hline Guarumo angustifolia & 3 & 0,161 & 2,206 & 0,735 & 1,620 & 0,540 & 3,826 & 1,275 \\
\hline
\end{tabular}




\begin{tabular}{|c|c|c|c|c|c|c|c|c|}
\hline Guarumo hoja blanca & 3 & 0,126 & 2,206 & 0,735 & 1,274 & 0,425 & 3,479 & 1,160 \\
\hline Habíocolorado & 3 & 0,153 & 2,206 & 0,735 & 1,546 & 0,515 & 3,751 & 1,250 \\
\hline Hintachi & 2 & 0,114 & 1,745 & 0,582 & 1,595 & 0,532 & 3,341 & 1,114 \\
\hline Laurel & 2 & 0,200 & 1,846 & 0,615 & 2,833 & 0,944 & 4,679 & 1,560 \\
\hline Malva & 15 & 1,418 & 12,157 & 4,052 & 16,181 & 5,394 & 28,337 & 9,446 \\
\hline Malvablanca & 9 & 0,476 & 9,091 & 3,030 & 7,549 & 2,516 & 16,640 & 5,547 \\
\hline María & 7 & 0,286 & 7,071 & 2,357 & 4,535 & 1,512 & 11,606 & 3,869 \\
\hline Membrillo & 1 & 0,038 & 1,010 & 0,337 & 0,601 & 0,200 & 1,611 & 0,537 \\
\hline Motilón & 2 & 0,294 & 1,745 & 0,582 & 3,448 & 1,149 & 5,193 & 1,731 \\
\hline Ocotealongifolia & 2 & 0,099 & 2,020 & 0,673 & 1,577 & 0,526 & 3,597 & 1,199 \\
\hline Ortiguillo & 2 & 0,121 & 1,471 & 0,490 & 1,219 & 0,406 & 2,690 & 0,897 \\
\hline Palo María & 14 & 0,673 & 10,294 & 3,431 & 6,794 & 2,265 & 17,088 & 5,696 \\
\hline Pigue & 91 & 6,095 & 92,646 & 30,882 & 99,863 & 32,288 & 192,508 & 64,169 \\
\hline Ponce & 44 & 3,594 & 36,026 & 12,009 & 41,790 & 13,930 & 77,816 & 25,939 \\
\hline Ponce peq. & 7 & 0,461 & 7,402 & 2,467 & 8,342 & 2,781 & 15,744 & 5,248 \\
\hline Pumamaqui & 1 & 0,130 & 1,010 & 0,337 & 2,069 & 0,690 & 3,079 & 1,026 \\
\hline Roble & 1 & 0,081 & 1,010 & 0,337 & 1,288 & 0,429 & 2,298 & 0,766 \\
\hline Sande & 1 & 0,179 & 0,735 & 0,245 & 1,807 & 0,602 & 2,542 & 0,847 \\
\hline Sangre de drago & 1 & 0,091 & 1,010 & 0,337 & 1,446 & 0,482 & 2,456 & 0,819 \\
\hline Sangre de gallina & 1 & 0,084 & 0,735 & 0,245 & 0,852 & 0,284 & 1,587 & 0,529 \\
\hline Sapote & 1 & 0,088 & 0,735 & 0,245 & 0,885 & 0,295 & 1,621 & 0,540 \\
\hline Tachuelo & 2 & 0,086 & 1,471 & 0,490 & 0,868 & 0,289 & 2,338 & 0,779 \\
\hline Uva de monte & 1 & 0,032 & 0,735 & 0,245 & 0,319 & 0,106 & 1,054 & 0,351 \\
\hline Yakucaspi blanco & 1 & 0,036 & 1,111 & 0,370 & 0,680 & 0,227 & 1,791 & 0,597 \\
\hline Yakucaspi colorado & 2 & 0,087 & 1,846 & 0,615 & 1,343 & 0,448 & 3,190 & 1,063 \\
\hline Yakucaspi de pantano & 3 & 0,135 & 2,206 & 0,735 & 1,360 & 0,453 & 3,565 & 1,188 \\
\hline Yutzo & 3 & 0,152 & 2,481 & 0,827 & 1,786 & 0,595 & 4,266 & 1,422 \\
\hline Yuyun & 1 & 0,075 & 1,010 & 0,337 & 1,188 & 0,396 & 2,198 & 0,733 \\
\hline Total general & 325,000 & 21,466 & 300,000 & 100,000 & 300,000 & 100,000 & 600,000 & 200,000 \\
\hline
\end{tabular}

Fuente: Investigación Directa

Elaboración: Carmen Luzuriaga 
Cuadro 8. Bosque Secundario Pendiente Alta

\begin{tabular}{|c|c|c|c|c|c|c|c|c|}
\hline NOMBRE COMÚN & Suma de F & $\begin{array}{l}\text { Suma de } \\
A B\end{array}$ & $\begin{array}{l}\text { Suma } \\
\text { de DR }\end{array}$ & $\%$ & $\begin{array}{l}\text { Suma } \\
\text { de DM }\end{array}$ & $\%$ & $\begin{array}{l}\text { Suma } \\
\text { de IVI }\end{array}$ & $\%$ \\
\hline ACHOTILLO & 3 & 0,189 & 1,390 & 0,463 & 1,293 & 0,431 & 2,684 & 0,895 \\
\hline ARRAYAN & 1 & 0,084 & 0,546 & 0,182 & 0,522 & 0,174 & 1,069 & 0,356 \\
\hline BALSA & 2 & 0,101 & 1,325 & 0,442 & 0,826 & 0,275 & 2,150 & 0,717 \\
\hline BALSA BLANCA & 9 & 0,458 & 3,797 & 1,266 & 3,398 & 1,133 & 7,195 & 2,398 \\
\hline BARBASCO & 2 & 0,809 & 1,093 & 0,364 & 5,006 & 1,669 & 6,099 & 2,033 \\
\hline CALUN CALUN & 1 & 0,115 & 0,546 & 0,182 & 0,709 & 0,236 & 1,255 & 0,418 \\
\hline CANELO & 1 & 0,034 & 0,546 & 0,182 & 0,208 & 0,069 & 0,754 & 0,251 \\
\hline CANELO AMARILLO & 2 & 0,128 & 1,093 & 0,364 & 0,791 & 0,264 & 1,883 & 0,628 \\
\hline CANELO BLANCO & 10 & 0,554 & 5,902 & 1,967 & 4,416 & 1,472 & 10,318 & 3,439 \\
\hline CANELO CAFÉ & 1 & 0,041 & 0,662 & 0,221 & 0,339 & 0,113 & 1,001 & 0,334 \\
\hline CANELO DE PANTANO & 5 & 0,296 & 2,732 & 0,911 & 1,830 & 0,610 & 4,562 & 1,521 \\
\hline \multicolumn{9}{|l|}{ CANELO HUESO DE } \\
\hline PESCADO & 2 & 0,122 & 1,325 & 0,442 & 1,005 & 0,335 & 2,329 & 0,776 \\
\hline CANELO NEGRO & 7 & 0,528 & 4,280 & 1,427 & 4,192 & 1,397 & 8,472 & 2,824 \\
\hline CAUCHO & 4 & 0,406 & 2,649 & 0,883 & 3,331 & 1,110 & 5,980 & 1,993 \\
\hline CEDAZO & 2 & 0,093 & 1,084 & 0,361 & 0,719 & 0,240 & 1,804 & 0,601 \\
\hline CEDRILLO & 14 & 1,224 & 7,615 & 2,538 & 8,857 & 2,952 & 16,472 & 5,491 \\
\hline CHICLE & 4 & 0,159 & 2,186 & 0,729 & 0,983 & 0,328 & 3,169 & 1,056 \\
\hline CHUNCHO BLANCO & 2 & 0,133 & 0,844 & 0,281 & 0,983 & 0,328 & 1,827 & 0,609 \\
\hline COLCA DE BP & 10 & 0,499 & 4,966 & 1,655 & 3,343 & 1,114 & 8,309 & 2,770 \\
\hline \multicolumn{9}{|l|}{ COLCA DE HOJA } \\
\hline BLANCA & 1 & 0,048 & 0,546 & 0,182 & 0,299 & 0,100 & 0,846 & 0,282 \\
\hline COLCA DE HOJA FINA & 1 & 0,041 & 0,422 & 0,141 & 0,306 & 0,102 & 0,728 & 0,243 \\
\hline COLCA HOJA FINA & 5 & 0,242 & 2,848 & 0,949 & 1,564 & 0,521 & 4,412 & 1,471 \\
\hline COPAL & 7 & 0,527 & 4,039 & 1,346 & 4,081 & 1,360 & 8,121 & 2,707 \\
\hline DONCEL & 2 & 0,144 & 1,093 & 0,364 & 0,892 & 0,297 & 1,985 & 0,662 \\
\hline DONCEL COCO & 2 & 0,174 & 1,209 & 0,403 & 1,260 & 0,420 & 2,469 & 0,823 \\
\hline \multicolumn{9}{|l|}{ DONCEL SANGRE DE } \\
\hline GAL & 1 & 0,033 & 0,422 & 0,141 & 0,242 & 0,081 & 0,664 & 0,221 \\
\hline DONCEL. & 1 & 0,385 & 0,546 & 0,182 & 2,382 & 0,794 & 2,929 & 0,976 \\
\hline DRAGO & 2 & 0,113 & 0,844 & 0,281 & 0,835 & 0,278 & 1,679 & 0,560 \\
\hline FALSO CAUCHO & 1 & 0,044 & 0,662 & 0,221 & 0,358 & 0,119 & 1,020 & 0,340 \\
\hline GUABA BEJUCA & 3 & 0,136 & 1,506 & 0,502 & 1,060 & 0,353 & 2,566 & 0,855 \\
\hline
\end{tabular}




\begin{tabular}{|c|c|c|c|c|c|c|c|c|}
\hline $\begin{array}{l}\text { GUABA BEJUCA DE } \\
\text { MONTE }\end{array}$ & 3 & 0,167 & 1,639 & 0,546 & 1,036 & 0,345 & 2,675 & 0,892 \\
\hline GUABA BP & 8 & 0,594 & 4,372 & 1,457 & 3,672 & 1,224 & 8,043 & 2,681 \\
\hline GUABA COLORADA & 1 & 0,258 & 0,546 & 0,182 & 1,595 & 0,532 & 2,141 & 0,714 \\
\hline GUABA DEL RIO & 23 & 1,539 & 13,032 & 4,344 & 9,935 & 3,312 & 22,967 & 7,656 \\
\hline GUABILLA & 21 & 1,620 & 13,186 & 4,395 & 13,137 & 4,379 & 26,324 & 8,775 \\
\hline GUAMBULA & 5 & 0,370 & 2,608 & 0,869 & 2,389 & 0,796 & 4,997 & 1,666 \\
\hline GUARANGO & 1 & 0,073 & 0,422 & 0,141 & 0,544 & 0,181 & 0,966 & 0,322 \\
\hline GUARANGO BLANCO & 2 & 0,500 & 1,093 & 0,364 & 3,095 & 1,032 & 4,187 & 1,396 \\
\hline $\begin{array}{l}\text { GUARANGO CON } \\
\text { ESPINO }\end{array}$ & 13 & 1,744 & 8,493 & 2,831 & 14,086 & 4,695 & 22,580 & 7,527 \\
\hline GUARANGO ROJO & 6 & 0,852 & 3,626 & 1,209 & 6,022 & 2,007 & 9,648 & 3,216 \\
\hline GUARUMO & 8 & 0,425 & 4,817 & 1,606 & 3,395 & 1,132 & 8,212 & 2,737 \\
\hline $\begin{array}{l}\text { GUARUMO DE HOJA } \\
\text { ROJA }\end{array}$ & 3 & 0,148 & 1,639 & 0,546 & 0,913 & 0,304 & 2,552 & 0,851 \\
\hline $\begin{array}{l}\text { GUARUMO HOJA } \\
\text { BLANCA }\end{array}$ & 4 & 0,327 & 2,186 & 0,729 & 2,024 & 0,675 & 4,210 & 1,403 \\
\hline GUAYABILLO & 3 & 0,129 & 1,639 & 0,546 & 0,798 & 0,266 & 2,438 & 0,813 \\
\hline HABIO & 3 & 0,297 & 1,639 & 0,546 & 1,836 & 0,612 & 3,475 & 1,158 \\
\hline HIGUERON & 1 & 0,296 & 0,662 & 0,221 & 2,433 & 0,811 & 3,095 & 1,032 \\
\hline HINTACHI & 9 & 0,409 & 4,038 & 1,346 & 3,063 & 1,021 & 7,101 & 2,367 \\
\hline JENJIBRE & 5 & 0,186 & 2,732 & 0,911 & 1,150 & 0,383 & 3,882 & 1,294 \\
\hline LAUREL & 1 & 0,073 & 0,662 & 0,221 & 0,602 & 0,201 & 1,264 & 0,421 \\
\hline MALVA & 24 & 1,646 & 10,740 & 3,580 & 12,039 & 4,013 & 22,779 & 7,593 \\
\hline MANZANO & 2 & 0,220 & 1,084 & 0,361 & 1,669 & 0,556 & 2,754 & 0,918 \\
\hline MARIA & 17 & 0,850 & 9,397 & 3,132 & 6,060 & 2,020 & 15,457 & 5,152 \\
\hline MATA PALO & 1 & 0,033 & 0,422 & 0,141 & 0,242 & 0,081 & 0,664 & 0,221 \\
\hline MELISO & 1 & 0,044 & 0,662 & 0,221 & 0,358 & 0,119 & 1,020 & 0,340 \\
\hline MEMBRILLO & 4 & 0,305 & 2,417 & 0,806 & 2,225 & 0,742 & 4,643 & 1,548 \\
\hline MENTOL & 1 & 0,055 & 0,546 & 0,182 & 0,339 & 0,113 & 0,886 & 0,295 \\
\hline MOTILON & 15 & 1,753 & 8,269 & 2,756 & 11,826 & 3,942 & 20,095 & 6,698 \\
\hline NEGRO CASPI & 1 & 0,063 & 0,662 & 0,221 & 0,517 & 0,172 & 1,180 & 0,393 \\
\hline ORTIGUILLO & 4 & 0,349 & 2,293 & 0,764 & 2,405 & 0,802 & 4,697 & 1,566 \\
\hline PALO DE ARDILLA & 3 & 0,286 & 1,987 & 0,662 & 2,345 & 0,782 & 4,332 & 1,444 \\
\hline PEPA DE ALGODON & 1 & 0,156 & 0,546 & 0,182 & 0,965 & 0,322 & 1,511 & 0,504 \\
\hline
\end{tabular}




\begin{tabular}{|c|c|c|c|c|c|c|c|c|}
\hline PEPA DE PAVA & 1 & 0,147 & 0,662 & 0,221 & 1,208 & 0,403 & 1,870 & 0,623 \\
\hline PIGUE & 158 & 8,523 & 68,366 & 22,789 & 63,368 & 21,123 & 131,734 & 43,911 \\
\hline PONCE & 63 & 5,581 & 35,995 & 11,998 & 38,861 & 12,954 & 74,856 & 24,952 \\
\hline ROBLE & 1 & 0,056 & 0,422 & 0,141 & 0,416 & 0,139 & 0,838 & 0,279 \\
\hline SAMDE & 1 & 0,113 & 0,422 & 0,141 & 0,835 & 0,278 & 1,257 & 0,419 \\
\hline SANDE & 3 & 0,566 & 1,755 & 0,585 & 4,245 & 1,415 & 6,000 & 2,000 \\
\hline SANGRE DE DRAGO & 5 & 0,318 & 3,080 & 1,027 & 2,361 & 0,787 & 5,440 & 1,813 \\
\hline SIN NOMBRE & 5 & 0,535 & 2,848 & 0,949 & 3,531 & 1,177 & 6,379 & 2,126 \\
\hline TACHUELO & 4 & 0,175 & 2,293 & 0,764 & 1,330 & 0,443 & 3,623 & 1,208 \\
\hline TAMBURO & 4 & 0,340 & 2,649 & 0,883 & 2,790 & 0,930 & 5,439 & 1,813 \\
\hline TUCUTA & 4 & 0,721 & 2,417 & 0,806 & 4,636 & 1,545 & 7,054 & 2,351 \\
\hline UVA DE MONTE & 3 & 0,292 & 1,755 & 0,585 & 1,902 & 0,634 & 3,658 & 1,219 \\
\hline YACU CASPI ROJO & 2 & 0,084 & 1,093 & 0,364 & 0,517 & 0,172 & 1,610 & 0,537 \\
\hline YACUCASPI & 1 & 0,035 & 0,662 & 0,221 & 0,285 & 0,095 & 0,947 & 0,316 \\
\hline \multicolumn{9}{|l|}{ YACUCASPI DE } \\
\hline PANTANO & 2 & 0,067 & 1,325 & 0,442 & 0,552 & 0,184 & 1,876 & 0,625 \\
\hline YaKU CASPI & 1 & 0,040 & 0,422 & 0,141 & 0,297 & 0,099 & 0,719 & 0,240 \\
\hline YUTZU & 11 & 0,529 & 7,053 & 2,351 & 4,122 & 1,374 & 11,175 & 3,725 \\
\hline TOTAL & & & & 100,000 & & 100,000 & & 200,000 \\
\hline
\end{tabular}

Fuente: Investigación Directa

Elaboración: Carmen Luzuriaga 
Cuadro 9. Regeneración natural en parcelas pendiente: alta, media y baja de Bosque Secundario.

\begin{tabular}{|c|c|c|c|c|}
\hline ESPECIE & PB & PM & PA & Maderable \\
\hline Agujon & 6 & 3 & 5 & \\
\hline Aguano & & 4 & 7 & B \\
\hline Arrayan & 2 & 5 & 5 & C \\
\hline Barbasco & 1 & & & \\
\hline Barbasco Caspi & & & 2 & \\
\hline Bateacaspi & 15 & & & C \\
\hline Canelo & 17 & 16 & 16 & $M$ \\
\hline CaneloAtuncholo & & 6 & & $M$ \\
\hline CaneloBobo & 8 & 2 & & $M$ \\
\hline Canelo Blanco & 3 & & & M \\
\hline Canelo Café & & 3 & & $M$ \\
\hline Carahuasca & & 2 & 7 & $M$ \\
\hline Cedro & 1 & 2 & & B \\
\hline Cedazo & & 7 & 2 & $M$ \\
\hline Ciruelo & 7 & 4 & 3 & $M$ \\
\hline Copal & 8 & 3 & 3 & B \\
\hline Colca & 14 & 12 & 16 & C \\
\hline Colca de hoja Fina & & 5 & & $\mathrm{C}$ \\
\hline Chontilla & & 3 & & \\
\hline Doncel & & 5 & 13 & B \\
\hline Guarango & 3 & 4 & & C \\
\hline Guarango colorado & & & 5 & C \\
\hline Guabilla & 10 & 11 & 6 & $\mathrm{M}$ \\
\hline Guaba del río & & 3 & & $M$ \\
\hline Guayabillo & 5 & 2 & 3 & C \\
\hline Helecho & & 10 & & \\
\hline María & & 5 & 4 & C \\
\hline
\end{tabular}




\begin{tabular}{|l|c|c|c|c|}
\hline Matico silvestre & & 7 & 3 & \\
\hline Membrillo & & & 3 & $\mathrm{M}$ \\
\hline Moradilla & 18 & 3 & & $\mathrm{C}$ \\
\hline Malaire & & 2 & 2 & \\
\hline Mindal & & 5 & 3 & $\mathrm{M}$ \\
\hline Motilon & 13 & 11 & 5 & $\mathrm{M}$ \\
\hline Moquillo & & 1 & 2 & $\mathrm{C}$ \\
\hline Negro Caspi & 2 & 3 & 2 & $\mathrm{C}$ \\
\hline Pepa de pava & 3 & & & $\mathrm{M}$ \\
\hline Ponce & & & 2 & $\mathrm{C}$ \\
\hline Pumamaqui & 3 & 3 & & $\mathrm{~B}$ \\
\hline Quilo & 12 & 8 & 5 & $\mathrm{C}$ \\
\hline Senna & & 2 & 1 & $\mathrm{C}$ \\
\hline Sin Nombre & & & 3 & \\
\hline Sarza parrilla & 2 & & & $\mathrm{C}$ \\
\hline Uva & 11 & & 3 & $\mathrm{M}$ \\
\hline Yacucaspi & 23 & 2 & 12 & $\mathrm{C}$ \\
\hline Yacucaspi colorado & & & 3 & $\mathrm{C}$ \\
\hline Yacucaspi de & & & & $\mathrm{C}$ \\
\hline pantano & & & & \\
\hline Yutzu & & & & \\
\hline Zanahoria & & & & \\
\hline Total & & & & \\
\hline Especies & & & & \\
\hline
\end{tabular}

Fuente: Investigación Directa

Elaboración: Carmen Luzuriaga 
Cuadro 10. Árboles con mayor regeneración en Bosque Secundario

\begin{tabular}{|l|c|r|}
\hline \multicolumn{1}{|c|}{ ESPECIE } & Maderable & \multicolumn{2}{|c|}{ Total } \\
\hline Agujon & & 14 \\
\hline Bateacaspi & C & 15 \\
\hline Canelo & M & 49 \\
\hline Ciruelo & M & 14 \\
\hline Copal & B & 14 \\
\hline Colca & C & 42 \\
\hline Doncel & B & 18 \\
\hline Guabilla & M & 27 \\
\hline Moradilla & C & 21 \\
\hline Motilon & M & 29 \\
\hline Quilo & C & 25 \\
\hline Uva & M & 14 \\
\hline Yacucaspi & C & 37 \\
\hline
\end{tabular}

Fuente: InvestigaciónDirecta

Elaboración: Carmen Luzuriaga
Cuadro 13. Pendiente Baja en Bosque Primario.

\begin{tabular}{|l|r|r|r|r|r|}
\hline NOMBRE COMÚN & $\mathrm{F}$ & $\mathrm{AB}\left(\mathrm{m}^{2}\right)$ & $\mathrm{DR}(\%)$ & $\mathrm{DM}(\%)$ & $\mathrm{IVI}(\%)$ \\
\hline ACHOTILLO & 1 & 0,11 & 0,76 & 1,08 & 1,84 \\
\hline ACHOTILLO MACHO & 1 & 0,03 & 0,76 & 0,34 & 1,10 \\
\hline BALSA & 1 & 0,03 & 0,76 & 0,31 & 1,07 \\
\hline CANELO BLANCO & 8 & 0,44 & 6,11 & 4,29 & 10,40 \\
\hline CAUCHO & 3 & 0,14 & 2,29 & 1,33 & 3,62 \\
\hline CEDRILLO & 2 & 0,18 & 1,53 & 1,75 & 3,27 \\
\hline COLCA DE HOJA ROJA & 3 & 0,21 & 2,29 & 2,06 & 4,35 \\
\hline COPAL & 1 & 0,72 & 0,76 & 6,98 & 7,74 \\
\hline DRAGO & 1 & 0,03 & 0,76 & 0,33 & 1,09 \\
\hline GUABA BEJUCA & 19 & 1,03 & 14,50 & 10,05 & 24,56 \\
\hline GUABILLA & 11 & 0,64 & 8,40 & 6,25 & 14,65 \\
\hline GUARANGO & 6 & 0,33 & 4,58 & 3,25 & 7,83 \\
\hline GUARUMO & 10 & 1,51 & 7,63 & 14,74 & 22,38 \\
\hline HABIO & 1 & 0,39 & 5,34 & 3,83 & 9,18 \\
\hline MALVA & 1 & 0,12 & 0,76 & 1,15 & 1,92 \\
\hline MATA PALO & 1 & 0,12 & 0,76 & 1,13 & 1,90 \\
\hline NEGRO CASPI & 1 & 0,15 & 0,76 & 1,50 & 2,26 \\
\hline PIGUE & & 0,32 & 0,76 & 3,10 & 3,86 \\
\hline PONCE & 1 & 39,69 & 36,07 & 75,77 \\
\hline UVA DE MONTE & 1 & 0,76 & 0,46 & 1,22 \\
\hline
\end{tabular}

Fuente: InvestigaciónDirecta

Elaboración: Carmen Luzuriaga 
Cuadro 14. Pendiente Media en Bosque Primario.

\begin{tabular}{|c|c|c|c|c|c|}
\hline NOMBRE COMÚN & $\mathbf{F}$ & $A B\left(m^{2}\right)$ & DR(\%) & DM(\%) & IVI(\%) \\
\hline ACHOTILLO MACHO & 1 & 0,05 & 0,60 & 0,28 & 0,88 \\
\hline APIO & 3 & 0,54 & 1,80 & 3,26 & 5,06 \\
\hline CANELO AMARILLO & 2 & 0,10 & 1,20 & 0,59 & 1,79 \\
\hline CANELO BLANCO & 7 & 0,47 & 4,19 & 2,86 & 7,06 \\
\hline CANELO CAFÉ & 2 & 0,10 & 1,20 & 0,62 & 1,82 \\
\hline CANELO HUESO DE PESCADO & 1 & 0,04 & 0,60 & 0,26 & 0,86 \\
\hline CARAHUASCA & 3 & 0,15 & 1,80 & 0,94 & 2,74 \\
\hline CARAHUASCA & 2 & 0,14 & 1,20 & 0,83 & 2,03 \\
\hline CAUCHO & 2 & 0,20 & 1,20 & 1,19 & 2,38 \\
\hline CEDAZO & 3 & 0,14 & 1,80 & 0,88 & 2,67 \\
\hline CEDRILLO & 7 & 0,83 & 4,19 & 5,05 & 9,24 \\
\hline CEDRO & 1 & 0,84 & 0,60 & 5,11 & 5,71 \\
\hline CEIBO & 1 & 0,97 & 0,60 & 5,92 & 6,52 \\
\hline CHICLE & 2 & 0,18 & 1,20 & 1,11 & 2,31 \\
\hline COLCA & 1 & 0,04 & 0,60 & 0,24 & 0,84 \\
\hline COPAL & 2 & 0,17 & 1,20 & 1,00 & 2,20 \\
\hline DONCEL & 1 & 0,32 & 0,60 & 1,93 & 2,53 \\
\hline FLOR AMARILLA & 3 & 0,34 & 1,80 & 2,07 & 3,86 \\
\hline GUABA DE RIO & 2 & 0,07 & 1,20 & 0,45 & 1,65 \\
\hline GUABILLA & 20 & 1,16 & 11,98 & 7,06 & 19,04 \\
\hline GUARANGO BLANCO & 2 & 0,13 & 1,20 & 0,79 & 1,98 \\
\hline GUARANGO ESPINUDO & 9 & 1,20 & 5,39 & 7,32 & 12,71 \\
\hline GUARUMO & 7 & 0,47 & 4,19 & 2,83 & 7,03 \\
\hline INTACHI & 2 & 0,10 & 1,20 & 0,59 & 1,79 \\
\hline JIGUA & 1 & 0,03 & 0,60 & 0,19 & 0,79 \\
\hline MANZANO & 2 & 0,63 & 1,20 & 3,84 & 5,04 \\
\hline MARIA & 1 & 0,04 & 0,60 & 0,24 & 0,84 \\
\hline MATA PALO & 4 & 0,68 & 2,40 & 4,12 & 6,51 \\
\hline MORAL & 2 & 0,19 & 1,20 & 1,13 & 2,32 \\
\hline MOTILON & 10 & 1,04 & 5,99 & 6,32 & 12,31 \\
\hline
\end{tabular}

Fuente: InvestigaciónDirecta

Elaboración: Carmen Luzuriaga
Cuadro 15. Pendiente Alta en Bosque Primario.

\begin{tabular}{|c|c|c|c|c|c|}
\hline NOMBRE COMÚN & $\mathbf{F}$ & $A B\left(m^{2}\right)$ & DR(\%) & DM(\%) & IVI(\%) \\
\hline CANELO BOBO & 3 & 0,23 & 2,31 & 2,01 & 4,32 \\
\hline GUARANGO & 8 & 0,70 & 6,15 & 6,25 & 12,40 \\
\hline MOTILON & 12 & 0,97 & 9,23 & 8,68 & 17,91 \\
\hline TAMBURO & 6 & 1,02 & 4,62 & 9,11 & 13,73 \\
\hline GUAYABILLO & 3 & 0,17 & 2,31 & 1,51 & 3,82 \\
\hline CANELO AMARILLO & 4 & 0,47 & 3,08 & 4,23 & 7,30 \\
\hline GUABILLA DE MONTE & 11 & 0,82 & 8,46 & 7,35 & 15,81 \\
\hline ORTIGUILLO & 4 & 0,27 & 3,08 & 2,46 & 5,53 \\
\hline CEDRILLO & 10 & 0,93 & 7,69 & 8,28 & 15,97 \\
\hline CHICLE & 2 & 0,16 & 1,54 & 1,39 & 2,93 \\
\hline MARIA & 2 & 0,15 & 1,54 & 1,34 & 2,88 \\
\hline PONCE & 16 & 1,28 & 12,31 & 11,46 & 23,77 \\
\hline YACUCASPI BLANCO & 2 & 0,15 & 1,54 & 1,34 & 2,88 \\
\hline CABO DE HACHA & 1 & 0,23 & 0,77 & 2,06 & 2,82 \\
\hline PALO DE ARDILLA & 3 & 0,22 & 2,31 & 2,01 & 4,32 \\
\hline DONCEL & 1 & 0,04 & 0,77 & 0,32 & 1,09 \\
\hline TUCUTA & 1 & 0,04 & 0,77 & 0,38 & 1,15 \\
\hline CEIBO & 3 & 0,40 & 2,31 & 3,61 & 5,92 \\
\hline GUARUMO & 1 & 0,04 & 0,77 & 0,40 & 1,17 \\
\hline CANELO BLANCO & 2 & 0,13 & 1,54 & 1,13 & 2,67 \\
\hline MEMBRILLO & 2 & 0,19 & 1,54 & 1,73 & 3,27 \\
\hline MANZANO & 2 & 0,27 & 1,54 & 2,37 & 3,91 \\
\hline PIGUE & 7 & 0,39 & 5,38 & 3,47 & 8,85 \\
\hline UVA DE MONTE & 1 & 0,06 & 0,77 & 0,56 & 1,33 \\
\hline MALVA & 2 & 0,09 & 1,54 & 0,84 & 2,38 \\
\hline MELLISO & 2 & 0,07 & 1,54 & 0,64 & 2,18 \\
\hline COLCA & 1 & 0,04 & 0,77 & 0,40 & 1,17 \\
\hline TACHUELO & 1 & 0,10 & 0,77 & 0,86 & 1,63 \\
\hline NEGRO CASPI & 2 & 0,11 & 1,54 & 0,98 & 2,52 \\
\hline CEDAZO & 1 & 0,12 & 0,77 & 1,06 & 1,83 \\
\hline
\end{tabular}

Fuente: Investigación Directa

Elaboración: Carmen Luzuriaga 
Cuadro 16. Regeneración Natural en Bosque Primario.

\begin{tabular}{|c|c|c|c|c|}
\hline ESPECIE & PA & PM & PB & MAD \\
\hline Aguano & 2 & 1 & & B \\
\hline Agujones & & & 4 & \\
\hline Arrayan & 21 & 10 & & C \\
\hline Bateacaspi & 2 & & & C \\
\hline Canelo & 10 & & 4 & C \\
\hline Caneloamarillo & & 3 & & B \\
\hline Chontilla & & & 3 & \\
\hline Ciruelo & & & 3 & \\
\hline Colca & 15 & 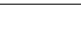 & 3 & C \\
\hline Colca de bosqueprimario & & 2 & & $M$ \\
\hline Copal & & 2 & 3 & B \\
\hline Doncel & & 3 & & B \\
\hline Guabilla & & 5 & 4 & $M$ \\
\hline Guarango & 1 & 10 & & C \\
\hline Guayabillo & & 1 & 3 & C \\
\hline Malaire & 1 & & 2 & \\
\hline Malaire & & 1 & & \\
\hline Maria & & 5 & & C \\
\hline Millizo & 2 & & & $M$ \\
\hline Moquillo & & 1 & 2 & \\
\hline Motilon & 4 & 4 & & $M$ \\
\hline Negro caspi & & 2 & 2 & C \\
\hline \multicolumn{5}{|l|}{ Pantano } \\
\hline Ponce & & & 3 & C \\
\hline Pumamaqui & & & 2 & \\
\hline Quilo & 2 & & & C \\
\hline Quilo & & 3 & & C \\
\hline Uva & 2 & 2 & 2 & \\
\hline Yacucaspi & 2 & 2 & & C \\
\hline Yacucaspi de & 2 & & & C \\
\hline
\end{tabular}

Fuente: InvestigaciónDirecta

Elaboración: Carmen Luzuriaga
Cuadro 17. Especies forestales maderables en el bosque

\begin{tabular}{|c|c|}
\hline ESPECIES & MADERA \\
\hline Achotillo & C \\
\hline Achotillo macho & C \\
\hline Aguano & B \\
\hline Arrayan & $\mathrm{C}$ \\
\hline Balsa blanca & $M$ \\
\hline Batea caspi & $\mathrm{C}$ \\
\hline Boya & $M$ \\
\hline Canelo & $M$ \\
\hline Canelo amarillo & $\mathrm{B}$ \\
\hline Canelo atuncholo & $M$ \\
\hline Caneloblanco & $M$ \\
\hline Canelobobo & $M$ \\
\hline Canelo café & $M$ \\
\hline Carahuasca & $M$ \\
\hline Cedazo & M \\
\hline Cedrillo & $\mathrm{C}$ \\
\hline Cedro & $\mathrm{B}$ \\
\hline Ciruelo & $M$ \\
\hline Colca & C \\
\hline Colcahojafina & $\mathrm{C}$ \\
\hline Copal & B \\
\hline Doncel & $\mathrm{B}$ \\
\hline Doncel coco & $\mathrm{M}$ \\
\hline Guaba del río & $\mathrm{C}$ \\
\hline Guabilla & $\mathrm{C}$ \\
\hline Guarango & $\mathrm{C}$ \\
\hline Guarango colorado & $\mathrm{C}$ \\
\hline Guayabillo & $\mathrm{C}$ \\
\hline Laurel & $\mathrm{B}$ \\
\hline Malva & $\mathrm{C}$ \\
\hline María & $\mathrm{C}$ \\
\hline Mellizo & $M$ \\
\hline Membrillo & $M$ \\
\hline
\end{tabular}




\begin{tabular}{|l|c|}
\hline Mindal & C \\
\hline Moradilla & C \\
\hline Motilón & M \\
\hline Negro caspi & C \\
\hline Ortiguillo & M \\
\hline Pepa de pava & M \\
\hline Pigue & C \\
\hline Ponce & $\mathrm{C}$ \\
\hline Pumamaqui & B \\
\hline Sande & M \\
\hline Sangre de gallina & M \\
\hline Sarzaparrilla & $\mathrm{C}$ \\
\hline Senna & $\mathrm{C}$ \\
\hline Tachuelo & $\mathrm{C}$ \\
\hline Tucuta & $\mathrm{M}$ \\
\hline Uva & $\mathrm{M}$ \\
\hline Yacucaspi de pantano & $\mathrm{C}$ \\
\hline Yacucaspi & $\mathrm{C}$ \\
\hline Yacucaspicolorado & $\mathrm{C}$ \\
\hline Yutzu & $\mathrm{M}$ \\
\hline
\end{tabular}

Fuente: InvestigaciónDirecta

Elaboración: Carmen Luzuriaga 
